\title{
Risk factors for gastric intraepithelial neoplasia in Chinese adults: a case-control study
}

This article was published in the following Dove Press journal: Cancer Management and Research

\section{Yanqiu Yu,' Cheng Fang, ${ }^{2}$ Chunyan Peng, 3,4 Shanshan Shen, ${ }^{4}$ Guifang $X u,{ }^{4}$ Qi Sun, ${ }^{5}$ Lin $\mathrm{Li}^{5}{ }^{5}$ Chuan Su, ${ }^{6}$ Xiaoping Zou ${ }^{3,4}$ \\ 'Department of Gastroenterology, The Affiliated Jiangning Hospital with Nanjing Medical University, Nanjing, People's Republic of China; \\ ${ }^{2}$ Department of Gastroenterology, The Second Affiliated Hospital of Zhejiang University School of Medicine, Hangzhou, People's Republic of China; ${ }^{3}$ Department of Gastroenterology, The Affiliated Drum Tower Clinical Medical School, Nanjing Medical University, Nanjing, People's Republic of China; ${ }^{4}$ Department of Gastroenterology, Nanjing Drum Tower Hospital, The Affiliated Hospital of Nanjing University Medical School, Nanjing, People's Republic of China; ${ }^{5}$ Department of Pathology, Nanjing Drum Tower Hospital, The Affiliated Hospital of Nanjing University Medical School, Nanjing, People's Republic of China; ${ }^{6}$ Department of Pathogen Biology \& Immunology, Jiangsu Key Laboratory of Pathogen Biology, Nanjing Medical University, Nanjing, People's Republic of China}

Correspondence: Chuan Su Department of Pathogen Biology \& Immunology, Jiangsu Key Laboratory of Pathogen Biology, Nanjing Medical University, No. I0I Longmian Road, Nanjing 2 I I 66, People's Republic of China Email chuansu@njmu.edu.cn

Xiaoping Zou

Department of Gastroenterology, The Affiliated Drum Tower Clinical Medical School, Nanjing Medical University, No. 32I Zhongshan Road, Nanjing 210008, People's Republic of China Email13770771661@I63.com
Background: Gastric carcinoma (GC) is the third most frequent malignancy and the second most common cancer-related cause of death cause worldwide. Gastric intraepithelial neoplasia (GIN) is a well-documented precancerous lesion of GC. In this case-control study, we comprehensively explored the clinical and pathological characteristics of GIN, with the aim to identify its potential risk factors.

Patients and methods: A total of 630 consecutive patients who underwent endoscopic submucosal dissection or mucosal resection for GIN were initially included. The detailed characteristics of all eligible patients and well-matched healthy controls were recorded and analyzed. Both univariate and multivariate logistic regression analyses were performed and presented with odds ratio (OR) and 95\% confidential interval (CI), with additional subgroup analyses based on lesion location.

Results: A total of 485 GIN-eligible patients were selected, among which 156 had proximal GIN. After follow-up, 434 patients with GIN and 310 age- and gender-matched healthy controls were included in the comparative analyses. Family cancer history (FCH); alcohol abuse; tobacco abuse; intake of high sodium, preserved food, spicy food, and less fruit; Helicobacter pylori (Hp) infection; and atrophic gastritis with intestinal metaplasia were more frequent in GIN patients. Thus, FCH (OR $=3.485,95 \%$ CI: 2.031-5.981), high sodium intake (OR $=2.830$, 95\% CI: $1.645-4.868$ ), less fruit intake (OR $=4.082,95 \% \mathrm{CI}: 2.515-6.625)$, Hp infection (OR $=2.307,95 \% \mathrm{CI}: 1.417-3.755)$, and atrophic gastritis with intestinal metaplasia $(\mathrm{OR}=15.070$, 95\% CI: 8.999-25.237) were independent risk factors for GIN. Further subgroup analyses demonstrated that the specific independent risk factor for proximal GIN was age (OR $=2.001$, 95\% CI: $1.003-3.994)$, whereas that for distal GIN was intake of high sodium (OR $=3.467$, 95\% CI: $1.896-6.338)$.

Conclusion: This study reported a comprehensive overview of the clinical and pathological characteristics of GIN. FCH, high sodium intake, less fruit intake, Hp infection, and atrophic gastritis were identified as the independent risk factors for GIN.

Keywords: gastric intraepithelial neoplasia, risk factor, case-control study, subgroup analysis

\section{Introduction}

Gastric carcinoma (GC) is the third most common malignancy worldwide with approximately 1.3 million newly diagnosed cases. ${ }^{1}$ In People's Republic of China, GC ranks as the second most frequently occurring cancer and cause of cancer deaths; approximately 679,100 new cases and 498,000 GC-related deaths were projected to occur in $2015 .^{2}$ The prognosis of GC differs significantly in accordance with various stages; the 5-year overall survival rate of early GC is over $90 \%$, while that of advanced 
GC is about $15 \% .{ }^{3}$ Early diagnosis and treatment of GC could contribute to the prolonged survival of GC patients. ${ }^{4}$

Gastric intraepithelial neoplasia (GIN) is well accepted as a precancerous lesion of GC, which can be divided into 2 categories according to lesion location: proximal and distal intraepithelial neoplasia (PGIN and DGIN, respectively) ${ }^{5}$ The annual incidence of GC is $6 \%$ for high-grade GIN patients within 5 years after diagnosis. ${ }^{6}$ To date, numerous efforts have been devoted to identify the clinical and epidemiological features of GC; $;^{7,8}$ however, GIN remains poorly understood. Furthermore, previous studies demonstrated that proximal and distal GC (PGC and DGC) are 2 distinct entities with different epidemiologic, clinicopathologic, and molecular biological features. ${ }^{9-11}$ Hence, it is of vital importance to explore the potential variety between risk factors for PGIN and DGIN.

In the current study, we retrospectively enrolled 485 GIN patients to provide a comprehensive overview of the clinical and pathological features of GIN. Furthermore, 310 age- and gender-matched healthy volunteers were recruited for comparison to identify the potential risk factors for GIN.

\section{Patients and methods}

\section{Patient selection}

From January 2007 to December 2014, 630 consecutive patients who underwent endoscopic submucosal dissection (ESD) or endoscopic mucosal resection (EMR) for GIN at Nanjing Drum Tower Hospital were initially included in the current study. The inclusion criteria were set as follows: 1) age $\geq 18$ years and 2) pathologically diagnosed with GIN after ESD/EMR by 2 independent experienced pathologists. The exclusion criteria were as follows: 1) pathologically diagnosed with GC after operation, 2) multifocal lesion which was defined as the distance between 2 lesions over $20 \mathrm{~mm}, 3$ ) a history of GC, and 4) tumor located at distal esophagus and gastroesophageal junction. The detailed selection criteria are demonstrated in Figure 1. A total of 485 patients with GIN were finally enrolled in this study. Furthermore, 310 gender- and age-matched outpatients who underwent endoscopy and biopsy confirmed to be negative for GC or GIN were recruited as the control group. The study protocol was approved by the institutional review board of Nanjing Drum Tower Hospital, and written informed consent was obtained from all individual participants.

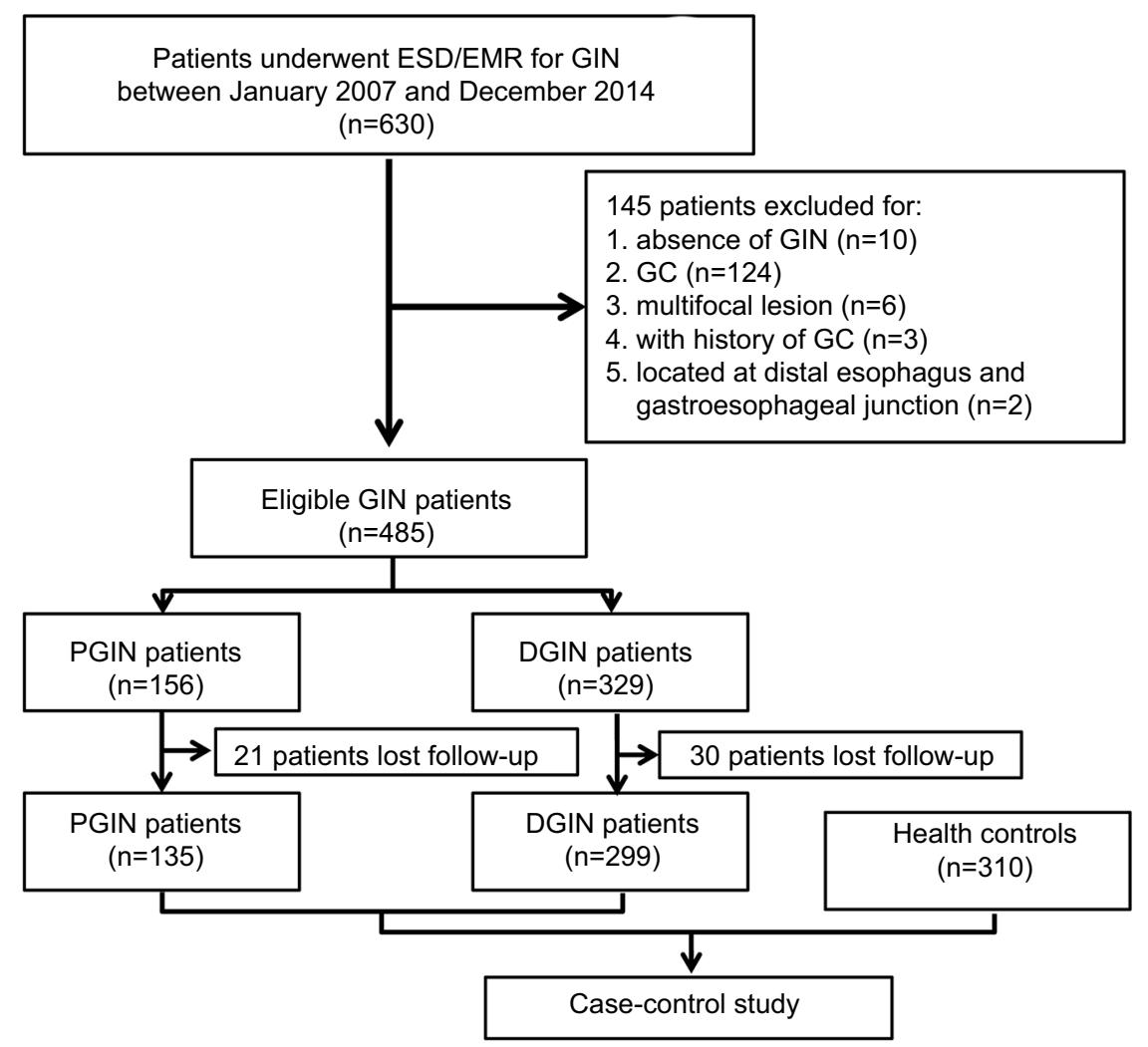

Figure I Study flow chart.

Notes: A total of 485 eligible GIN patients were included in this study, among which 434 patients with detailed follow-up information were compared with 3 I 0 well-matched controls.

Abbreviations: EMR, endoscopic mucosal resection; ESD, endoscopic submucosal dissection; GC, gastric carcinoma; GIN, gastric intraepithelial neoplasia; DGIN, distal GIN; PGIN, proximal GIN. 


\section{Study procedure}

All endoscopy procedures including ESD/EMR were performed by well-trained physicians. The endoscopic morphology of superficial lesions was recoded according to the Paris classification system. ${ }^{12}$ The representative histologic images of low- and high-grade GIN are shown in Figure 2A and B, respectively. Furthermore, the GIN lesions were categorized into 2 groups: PGIN, which was defined as a lesion located within 30 $\mathrm{mm}$ from the gastroesophageal junction and DGIN, which was described as a lesion located in the remaining regions. The electronic medical records were thoroughly reviewed, and clinical follow-up was performed by office visit or telephone contact. Information, including history of cancer, personal history, dietary habit, and present relevant diseases, were collected in a uniform form as was previously reported. ${ }^{13}$ Table S1 presents the detailed items and corresponding definitions.

\section{Statistical analysis}

The continuous variables are presented as mean $\pm \mathrm{SD}$ after the confirmation of normal distribution and were compared using Student's $t$-test or 1-way analysis of variance test. The categorical variables are presented as counts and percentages and compared with $\chi^{2}$ statistics or Fisher's exact test when appropriate. To identify the potential risk factors, the univariate and multivariate logistic regression analyses were applied and are presented as odds ratio (OR) with the corresponding $95 \%$ confidential interval $(95 \% \mathrm{CI})$. The statistical analyses were performed using SPSS (version 22.0; IBM Corporation, Armonk, NY, USA), and statistical significance was taken as a 2 -sided $P$-value $<0.05$.

\section{Results}

\section{Baseline clinical and pathological characteristics}

A total of 485 GIN patients were enrolled in this study. Among these participants, 156 were diagnosed with PGIN (Figure 2C). The proportion of incident PGIN cases increased gradually from $13.6 \%$ in $2007-2008$ to $35.3 \%$ in 2012-2014 (Figure 2D). The mean age was 62.3 years and differed between 2 groups (PGIN vs DGIN: $63.8 \pm 8.5$ vs
A

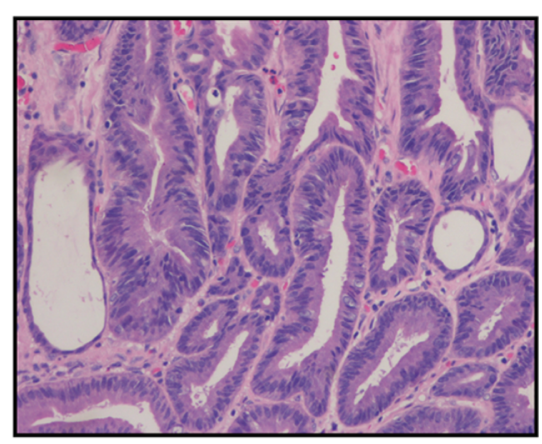

C

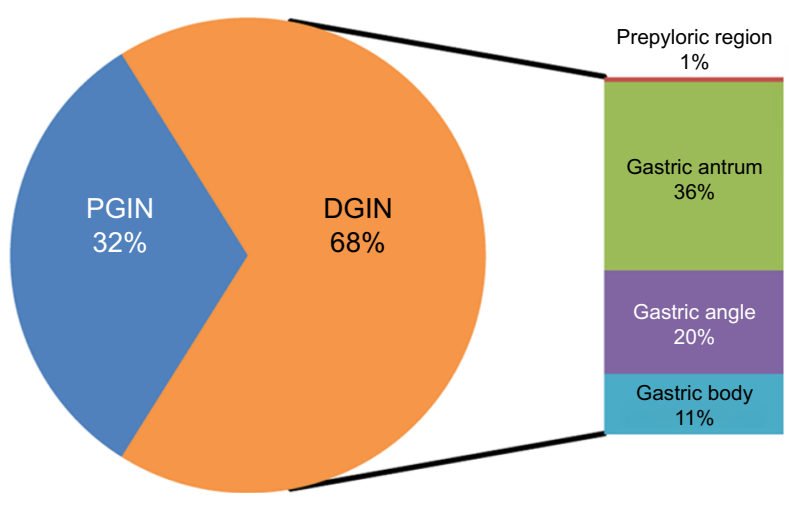

B
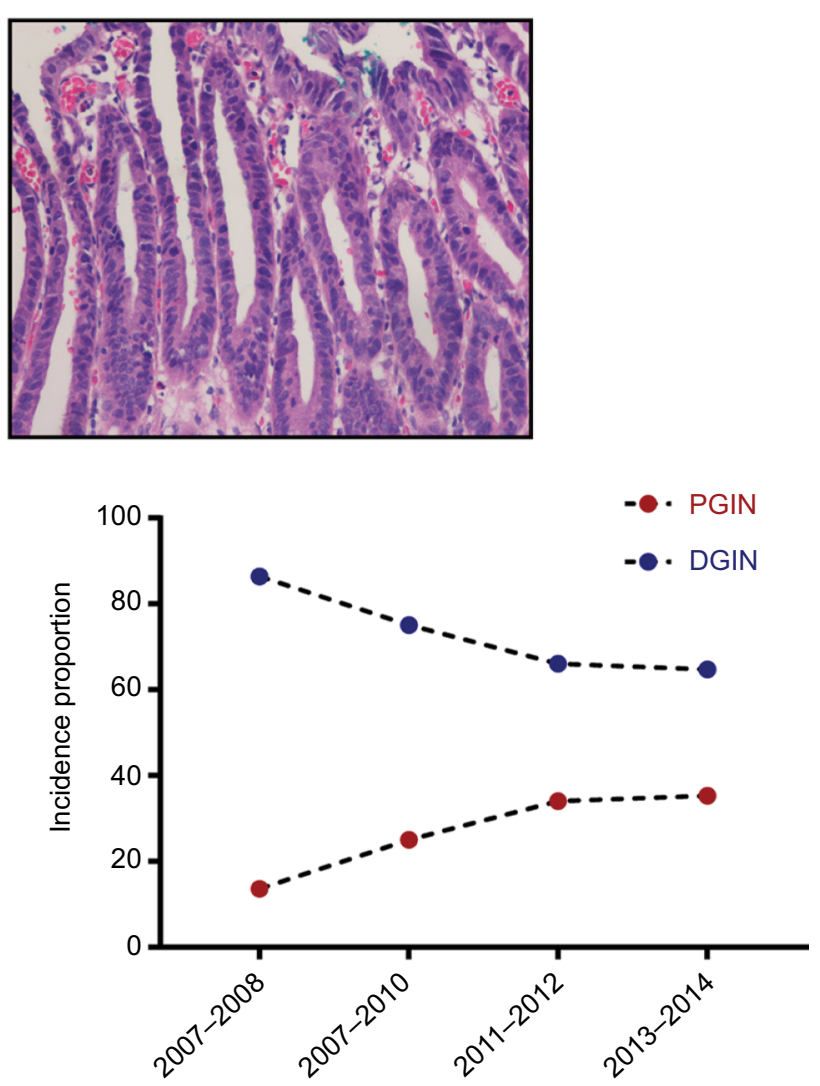

Figure 2 Overview of GIN distribution.

Notes: Representative histological images of low-grade intraepithelial neoplasia (A); high-grade intraepithelial neoplasia (B); location distribution of GIN (C); and proportion of 2 GIN types (D). (A, B) Magnification was set at $\times 200$.

Abbreviations: GIN, gastric intraepithelial neoplasia; DGIN, distal GIN; PGIN, proximal GIN. 
61.6 \pm 9.4 years, $P=0.018$; Table 1 ). As summarized in Table 1 , significant differences were observed in the distributions of age, gender, endoscopic morphology, histologic grade, occurrence of gastritis cystica profunda, and pancreatic metaplasia between PGIN and DGIN patients. During the follow-up period, $51(10.5 \%)$ were patients lost to followup, and the remaining 434 GIN patients with 310 age- and gender-matched healthy controls were included in the comparative analyses (Figure 1).

\section{Comparison of risk factors for GIN and subgroup analyses stratified by PGIN and DGIN}

Compared to the control group, family cancer history (FCH); alcohol abuse; tobacco abuse; intake of high sodium, preserved food, spicy food, less fruit; Helicobacter pylori (Hp) infection, and atrophic gastritis with intestinal metaplasia were more frequent in GIN patients (Table 2). Further stratification analyses revealed the similar results in both groups, except that the number of patients with PGIN aged over 60 years was higher than that of patients with DGIN $(P=0.001$, Table 2).

\section{Identification of risk factors for GIN}

To identify potential risk factors for GIN, univariate logistic regression analyses were conducted, and it was found that FCH; alcohol abuse; tobacco abuse; intake of high sodium, preserved food, spicy food, and less fruit; Hp infection; and atrophic gastritis with intestinal metaplasia were found as potential risk factors for GIN (Table 3). The subgroup analyses showed that age ( $\geq 60$ years) was a high risk for PGIN (OR $=2.265,95 \%$ CI: $1.455-3.524, P=0.001)$ than for DGIN (OR $=1.348,95 \%$ CI: $0.975-1.864, P=0.070)$.

Subsequently, multivariate logistic regression analyses were performed (Table 4). FCH, high sodium intake, less fruit intake, Hp infection, and atrophic gastritis with intestinal metaplasia were identified as independent risk factors for GIN. Further stratification analyses confirmed that FCH, less fruit intake, Hp infection, and atrophic gastritis with intestinal metaplasia were independent risk factors for both PGIN and

Table I Clinical and pathological characteristics of patients with GIN

\begin{tabular}{|c|c|c|c|c|}
\hline Variables & GIN $(n=485)$ & PGIN $(n=156)$ & DGIN (n=329) & $P$-value \\
\hline \multicolumn{5}{|l|}{ Age (years) } \\
\hline Mean \pm SD & $62.3 \pm 9.2$ & $63.8 \pm 8.5$ & $61.6 \pm 9.4$ & 0.018 \\
\hline$\leq 40$ & $3(0.6 \%)$ & I (0.6\%) & $2(0.6 \%)$ & 0.048 \\
\hline $4 I-50$ & $52(10.7 \%)$ & $10(6.4 \%)$ & $42(12.8 \%)$ & \\
\hline $5 I-60$ & $147(30.3 \%)$ & 40 (25.7\%) & $107(32.5 \%)$ & \\
\hline $6 \mathrm{I}-70$ & $188(38.8 \%)$ & $73(46.8 \%)$ & 115 (35\%) & \\
\hline$\geq 71$ & 95 (19.6\%) & $32(20.5 \%)$ & 63 (19.1\%) & \\
\hline \multicolumn{5}{|l|}{ Gender } \\
\hline Male & 367 (75.7\%) & $13 \mid(84.0 \%)$ & $236(71.7 \%)$ & 0.003 \\
\hline Female & II 8 (24.3\%) & $25(16.0 \%)$ & $93(28.3 \%)$ & \\
\hline \multicolumn{5}{|l|}{ Endoscopic morphology } \\
\hline I & $183(37.7 \%)$ & $48(30.8 \%)$ & 135 (41.0\%) & 0.001 \\
\hline lla & 81 (6.7\%) & $16(10.3 \%)$ & 65 (19.7\%) & \\
\hline Ilb & $72(14.8 \%)$ & 29 (I8.5\%) & 43 (13.1\%) & \\
\hline Ilc & $122(25.2 \%)$ & $5 \mathrm{I}(32.7 \%)$ & $7 \mathrm{I}(2 \mathrm{I} .6 \%)$ & \\
\hline III & $27(5.6 \%)$ & $12(7.7 \%)$ & $15(4.6 \%)$ & \\
\hline \multicolumn{5}{|l|}{ Lesion size $(\mathrm{cm})$} \\
\hline Mean \pm SD & $2.6 \pm I .1$ & $2.5 \pm 1.3$ & $2.7 \pm 1.2$ & 0.369 \\
\hline \multicolumn{5}{|l|}{ Histological grade } \\
\hline Low grade & 237 (48.9\%) & $57(36.5 \%)$ & $180(54.7 \%)$ & $<0.001$ \\
\hline High grade & $248(51.1 \%)$ & 99 (63.5\%) & 149 (45.3\%) & \\
\hline \multicolumn{5}{|c|}{ Surrounding mucosa manifestation } \\
\hline Chronic gastritis & $478(98.6 \%)$ & $154(98.7 \%)$ & $324(98.5 \%)$ & 0.838 \\
\hline Intestinal metaplasia & 434 (89.5\%) & $134(85.9 \%)$ & 300 (91.2\%) & 0.076 \\
\hline Mucosal atrophy & 431 (88.9\%) & $133(85.3 \%)$ & $298(90.6 \%)$ & 0.082 \\
\hline Hp infection & $262(54.0 \%)$ & $75(48.1 \%)$ & $187(56.8 \%)$ & 0.071 \\
\hline Pancreatic metaplasia & $3(0.61 \%)$ & $3(1.3 \%)$ & $0(0 \%)$ & 0.033 \\
\hline Gastritis cystica profunda & $33(6.8 \%)$ & $24(I 5.4 \%)$ & $9(2.7 \%)$ & $<0.001$ \\
\hline
\end{tabular}

Abbreviations: GIN, gastric intraepithelial neoplasia; PGIN, proximal GIN; DGIN, distal GIN; Hp, Helicobacter pylori. 
Table 2 Comparison of subject characteristics between groups

\begin{tabular}{|c|c|c|c|c|c|c|c|}
\hline \multirow[t]{2}{*}{ Variables } & \multirow{2}{*}{$\begin{array}{l}\text { Control }(n=3 \mid 0) \\
\text { Data }\end{array}$} & \multicolumn{2}{|c|}{ GIN $(n=434)$} & \multicolumn{2}{|c|}{$\operatorname{PGIN}(n=\mid 35)$} & \multicolumn{2}{|c|}{ DGIN (n=299) } \\
\hline & & Data & $P$-value & Data & $P$-value & Data & $P$-value \\
\hline \multicolumn{8}{|l|}{ Age (years) } \\
\hline Mean \pm SD & $61.25 \pm 10.80$ & $62.02 \pm 9.03$ & 0.308 & $63.07 \pm 8.45$ & 0.167 & $61.77 \pm 9.28$ & 0.526 \\
\hline$<60$ & 140 (45.2\%) & I5I (34.8\%) & 0.098 & 37 (27.4\%) & 0.001 & 114 (38.1\%) & 0.070 \\
\hline$\geq 60$ & 170 (54.8\%) & 386 (65.2\%) & & 98 (72.6\%) & & 185 (6I.9\%) & \\
\hline \multicolumn{8}{|l|}{ Gender } \\
\hline Male & $215(69.4 \%)$ & 325 (74.9\%) & 0.096 & 103 (76.3\%) & 0.136 & 222 (74.2\%) & 0.180 \\
\hline Female & 95 (30.6\%) & 109 (25.1\%) & & 32 (23.7\%) & & 77 (25.8\%) & \\
\hline \multicolumn{8}{|l|}{ Hp infection } \\
\hline Positive & $93(30.0 \%)$ & 233 (53.7\%) & $<0.001$ & $6 \mathrm{I}(45.2 \%)$ & $<0.001$ & I 72 (57.5\%) & 0.001 \\
\hline Negative & 216 (69.7\%) & 201 (46.3\%) & & 74 (54.8\%) & & 127 (42.5\%) & \\
\hline \multicolumn{8}{|l|}{ BMI } \\
\hline Average \pm SD & $23.51 \pm 2.87$ & $23.69 \pm 3.37$ & 0.459 & $24.02 \pm 3.17$ & 0.129 & $23.57 \pm 3.45$ & 0.821 \\
\hline $\mathrm{BMI}<18.5$ & 14 (4.5\%) & $18(2.8 \%)$ & 0.171 & $6(4.5 \%)$ & 0.561 & $12(4.0 \%)$ & 0.165 \\
\hline $\mid 8.5 \leq \mathrm{BMI} \leq 24.0$ & 187 (60.3\%) & 230 (47.2\%) & & 72 (53.3\%) & & I 58 (52.8\%) & \\
\hline $24.0<\mathrm{BMI} \leq 28.0$ & 97 (3I.3\%) & 160 (36.2\%) & & $5 \mathrm{I}(37.8 \%)$ & & 109 (36.5\%) & \\
\hline $\mathrm{BMI}>28.0$ & $12(3.9 \%)$ & $26(6.7 \%)$ & & $6(4.4 \%)$ & & $20(6.7 \%)$ & \\
\hline \multicolumn{8}{|l|}{ History of cancer } \\
\hline Personal & $6(1.9 \%)$ & $8(1.8 \%)$ & 0.927 & I (0.7\%) & 0.352 & $7(2.3 \%)$ & 0.729 \\
\hline Family & 55 (17.7\%) & $187(43.1 \%)$ & $<0.001$ & $52(38.5 \%)$ & $<0.001$ & I 35 (45.2\%) & $<0.001$ \\
\hline \multicolumn{8}{|l|}{ Personal history } \\
\hline ETE & $51(16.5 \%)$ & $60(13.8 \%)$ & 0.321 & 15 (II.I\%) & 0.145 & 45 (I5.1\%) & 0.635 \\
\hline Use of NSAIDs & $18(5.8 \%)$ & 28 (6.5\%) & 0.719 & II (8.1\%) & 0.358 & $17(5.7 \%)$ & 0.949 \\
\hline Tobacco abuse & $115(37.1 \%)$ & 247 (56.9\%) & $<0.001$ & $76(56.3 \%)$ & $<0.001$ & I7| (57.2\%) & $<0.001$ \\
\hline Alcohol abuse & 114 (36.8\%) & 234 (53.9\%) & $<0.001$ & $72(53.3 \%)$ & $<0.001$ & 162 (54.2\%) & $<0.001$ \\
\hline \multicolumn{8}{|l|}{ Dietary habit } \\
\hline High sodium & $132(42.6 \%)$ & 338 (77.9\%) & $<0.001$ & 97 (71.9\%) & $<0.001$ & $24 \mid(80.6 \%)$ & $<0.001$ \\
\hline Preserved food & $83(26.9 \%)$ & 234 (53.9\%) & $<0.001$ & $67(49.6 \%)$ & $<0.001$ & 167 (55.9\%) & $<0.001$ \\
\hline Spicy food & $53(17.1 \%)$ & 173 (39.9\%) & $<0.001$ & 49 (36.3\%) & 0.010 & 124 (4I.5\%) & $<0.001$ \\
\hline Smoked food & $8(2.6 \%)$ & $13(3.0 \%)$ & 0.690 & $3(2.2 \%)$ & 0.472 & $10(3.3 \%)$ & 0.888 \\
\hline Fried food & 14 (4.5\%) & $38(8.8 \%)$ & 0.259 & $10(7.4 \%)$ & 0.671 & $28(9.4 \%)$ & 0.195 \\
\hline Hot food & 74 (23.9\%) & 172 (39.6\%) & 0.106 & 57 (42.2\%) & 0.085 & 115 (38.5\%) & 0.215 \\
\hline Less fruit & 99 (31.9\%) & 297 (68.4\%) & $<0.001$ & 96 (7I.I\%) & $<0.001$ & 201 (67.2\%) & $<0.001$ \\
\hline Less vegetable & 45 (I4.5\%) & $55(12.7 \%)$ & 0.467 & $14(10.4 \%)$ & 0.236 & $4 \mathrm{I}(13.7 \%)$ & 0.776 \\
\hline \multicolumn{8}{|l|}{ Present relevant illness } \\
\hline Anxiety/depression status & $68(21.9 \%)$ & $108(24.9 \%)$ & 0.133 & $36(26.7 \%)$ & 0.455 & $72(24.1 \%)$ & 0.109 \\
\hline Hypertension & $62(20.0 \%)$ & $108(24.9 \%)$ & 0.118 & $36(26.7 \%)$ & 0.119 & $72(24.0 \%)$ & 0.224 \\
\hline Diabetes mellitus & $22(7.1 \%)$ & $43(9.9 \%)$ & 0.181 & II (8.1\%) & 0.697 & $32(10.7 \%)$ & 0.118 \\
\hline GERD & 99 (31.9\%) & $120(27.7 \%)$ & 0.206 & $4 \mathrm{I}(30.4 \%)$ & 0.215 & 79 (26.4\%) & 0.135 \\
\hline Hiatal hernia & $3(1.0 \%)$ & $3(0.7 \%)$ & 0.678 & $2(1.5 \%)$ & 0.636 & $\mathrm{I}(0.3 \%)$ & 0.333 \\
\hline Columnar-lined esophagus & $2(0.6 \%)$ & $9(2.1 \%)$ & 0.111 & $3(2.2 \%)$ & 0.144 & $6(2.0 \%)$ & 0.140 \\
\hline $\begin{array}{l}\text { Gastric intestinal metaplasia and } \\
\text { atrophy }\end{array}$ & $112(36.1 \%)$ & 375 (86.4\%) & $<0.001$ & $122(90.4 \%)$ & $<0.001$ & $253(84.6 \%)$ & $<0.001$ \\
\hline
\end{tabular}

Abbreviations: GIN, gastric intraepithelial neoplasia; PGIN, proximal GIN; DGIN, distal GIN; Hp, Helicobacter pylori; BMI, body mass index; ETE, environmental toxin exposure; NSAIDs, nonsteroidal anti-inflammatory drugs; GERD, gastroesophageal reflux disease.

DGIN. One specific independent risk factor for PGIN was age, while that for DGIN was intake of high sodium.

\section{Discussion}

GC is a major public health problem globally, ${ }^{14,15}$ and GIN has been well accepted as the premalignant lesion of GC. ${ }^{3,16}$ In the present study, we comprehensively explored the clinical and pathological characteristics of GIN in the Chinese population.
The findings were as follows: 1) PGIN had half the number incidences of DGIN, but exhibited increasing trends during the study period, 2) age, FCH, less fruit intake, Hp infection, and atrophic gastritis with intestinal metaplasia were independent risk factors for GIN, and 3) age was a specific independent risk factor for PGIN, whereas that for DGIN was high sodium intake.

Emerging evidence indicated that GC could be divided into 2 categories, namely, PGC and DGC, due to different 
Table 3 Univariate analysis of risk factors for early PGIN and DGIN

\begin{tabular}{|c|c|c|c|c|}
\hline \multirow[t]{2}{*}{ Variables } & \multicolumn{2}{|l|}{ PGIN $(n=135)$} & \multicolumn{2}{|l|}{ DGIN (n=299) } \\
\hline & OR (95\% Cl) & $P$-value & OR (95\% Cl) & $P$-value \\
\hline \multicolumn{5}{|l|}{ Age (years) } \\
\hline$<60$ & I.000 (Reference) & & I.000 (Reference) & \\
\hline$\geq 60$ & $2.265(1.455-3.524)$ & 0.001 & $1.348(0.975-1.864)$ & 0.070 \\
\hline \multicolumn{5}{|l|}{ Gender } \\
\hline Female & I.000 (Reference) & & I.000 (Reference) & \\
\hline Male & $1.422(0.894-2.263)$ & 0.137 & $1.274(0.894-1.816)$ & 0.180 \\
\hline Hp infection & $3.665(2.347-5.722)$ & $<0.001$ & $2.513(1.778-3.552)$ & $<0.001$ \\
\hline \multicolumn{5}{|l|}{ BMI } \\
\hline $\mid 8.5 \leq \mathrm{BMI} \leq 24.0$ & I.000 (Reference) & & I.000 (Reference) & \\
\hline $\mathrm{BMI}<18.5$ & $\mathrm{I} .207(0.360-4.05 \mathrm{I})$ & 0.760 & $1.172(0.44 I-3.116)$ & 0.750 \\
\hline $24.0<\mathrm{BMI} \leq 28.0$ & $1.428(0.920-2.217)$ & 0.112 & $1.44 \mid(1.012-2.052)$ & 0.143 \\
\hline $\mathrm{BMI}>28.0$ & $1.358(0.490-3.764)$ & 0.556 & $1.528(1.216-5.257)$ & 0.437 \\
\hline \multicolumn{5}{|l|}{ History of cancer } \\
\hline Personal & $0.378(0.045-3 .|7|)$ & 0.352 & $1.215(0.403-3.657)$ & 0.730 \\
\hline Family & $2.905(1.847-4.568)$ & $<0.001$ & $3.817(2.637-5.524)$ & $<0.001$ \\
\hline \multicolumn{5}{|l|}{ Personal history } \\
\hline ETE & $0.635(0.343-1.174)$ & 0.148 & $0.900(0.58 I-I .392)$ & 0.635 \\
\hline Use of NSAIDs & $1.439(0.660-3.136)$ & 0.360 & $0.978(0.494-1.936)$ & 0.949 \\
\hline Tobacco abuse & $2.184(1.448-3.294)$ & $<0.001$ & $2.265(1.637-3.135)$ & $<0.001$ \\
\hline Alcohol abuse & 1.965 (1.305-2.959) & 0.001 & $2.033(1.470-2.81 \mathrm{I})$ & $<0.001$ \\
\hline \multicolumn{5}{|l|}{ Dietary habit } \\
\hline High sodium & $3.442(2.223-5.331)$ & $<0.001$ & $5.603(3.892-8.067)$ & $<0.001$ \\
\hline Preserved food & $2.683(1.76 I-4.087)$ & $<0.001$ & $3.450(2.458-4.843)$ & $<0.001$ \\
\hline Spicy food & $1.838(1.152-2.932)$ & 0.011 & $2.286(1.556-3.358)$ & $<0.001$ \\
\hline Smoked food & $0.614(0.160-2.354)$ & 0.477 & $0.934(0.363-2.407)$ & 0.888 \\
\hline Fried food & $1.200(0.5 \mid 7-2.783)$ & 0.671 & $1.550(0.796-3.018)$ & 0.198 \\
\hline Hot food & I.47I (0.947-2.287) & 0.086 & $1.258(0.875-1.810)$ & 0.215 \\
\hline Less fruit & $5.246(3.37 I-8.164)$ & $<0.001$ & $4.000(2.860-5.593)$ & $<0.001$ \\
\hline Less vegetable & $0.681(0.360-1.289)$ & 0.238 & $0.936(0.593-1.477)$ & 0.776 \\
\hline \multicolumn{5}{|l|}{ Present relevant illness } \\
\hline Anxiety/depression status & $0.834(0.518-1.343)$ & 0.456 & $0.728(0.493-1.074)$ & 0.109 \\
\hline Hypertension & I.43। (0.82-2.54) & 0.119 & $1.242(0.78-\mid .5 I)$ & 0.224 \\
\hline Diabetes mellitus & I.16I (0.546-2.468) & 0.697 & $1.569(0.889-2.768)$ & 0.120 \\
\hline GERD & $0.77 \mid(0.500-1.190)$ & 0.215 & $0.765(0.539-1.087)$ & 0.135 \\
\hline Hiatal hernia & $1.539(0.254-9.316)$ & 0.639 & $0.343(0.036-3.320)$ & 0.356 \\
\hline Columnar-lined esophagus & $3.527(0.582-21.353)$ & 0.170 & $3.154(0.631-15.750)$ & 0.162 \\
\hline Gastric intestinal metaplasia and atrophy & $16.59 \mid(8.952-30.748)$ & $<0.001$ & $9.723(6.581-\mid 4.366)$ & $<0.001$ \\
\hline
\end{tabular}

Abbreviations: PGIN, proximal gastric intraepithelial neoplasia; DGIN, distal gastric intraepithelial neoplasia; OR, odds ratio; 95\% CI, 95\% confidential interval; $\mathrm{HP}$, Helicobacter pylori; BMI, body mass index; ETE, environmental toxin exposure; NSAIDs, nonsteroidal anti-inflammatory drugs; GERD, gastroesophageal reflux disease.

epidemiologic, clinicopathologic, and molecular biological characteristics. ${ }^{9,10,17,18}$ However, recent studies challenged this classification because they found that PGC was more like DGC rather than esophageal adenocarcinoma. ${ }^{19-21}$ Considering the existing controversy about PGC and DGC, the current study was conducted to comprehensively explore the characteristics of GC's precancerous lesion, ie, GIN, and compare the difference between PGIN and DGIN. Among all 485 patients with GIN, 329 were diagnosed with DGIN and accounted for $67.8 \%$ of the population; a similar proportion of DGC was found in all GC cases. ${ }^{13,22}$ Furthermore, our results showed an increasing trend for PGIN as its proportion increased gradually during the study period 2007-2014, which was similar to the trends for PGC. ${ }^{23-25}$

To identify risk factors for GIN, a total of 434 GIN patients with 310 well-matched controls were recruited. Based on the results of logistic regression analyses, $\mathrm{FCH}$, high sodium, less fruit intake, Hp infection, and atrophic gastritis with intestinal metaplasia were identified as independent risk factors for GIN. All abovementioned parameters except $\mathrm{Hp}$ infection were also well documented as the risk factors for GC, which can support the conclusion that GIN 
Table 4 Multivariate analysis of risk factors for PGIN and DGIN

\begin{tabular}{|c|c|c|c|c|c|c|}
\hline \multirow[t]{2}{*}{ Variables } & \multicolumn{2}{|l|}{ GIN $(n=434)$} & \multicolumn{2}{|l|}{ PGIN $(n=\mid 35)$} & \multicolumn{2}{|l|}{ DGIN (n=299) } \\
\hline & OR (95\% Cl) & $P$-value & OR (95\% Cl) & $P$-value & OR (95\% Cl) & $P$-value \\
\hline \multicolumn{7}{|l|}{ Age (years) } \\
\hline$<60$ & I.000 (Reference) & & 1.000 (Reference) & & I.000 (Reference) & \\
\hline$\geq 60$ & $0.845(0.623-1.254)$ & 0.087 & 2.001 (1.003-3.994) & 0.049 & I.5II (0.895-2.549) & 0.122 \\
\hline Hp infection & 2.307 (I.4I7-3.755) & 0.001 & $2.057(1.60 I-5.838)$ & 0.003 & $2.553(I .393-4.68 I)$ & 0.002 \\
\hline Family history of cancer & $3.485(2.03 I-5.98 I)$ & $<0.001$ & $3.089(1.515-6.300)$ & 0.002 & $3.802(2.118-6.825)$ & $<0.001$ \\
\hline \multicolumn{7}{|l|}{ Personal history } \\
\hline Tobacco abuse & 1.561 (0.883-2.759) & 0.126 & $1.840(0.850-3.987)$ & 0.122 & $1.389(0.754-2.558)$ & 0.292 \\
\hline Alcohol abuse & $0.896(0.500-1.603)$ & 0.711 & $0.872(0.396-1.923)$ & 0.735 & $0.937(0.502-1.748)$ & 0.838 \\
\hline \multicolumn{7}{|l|}{ Dietary habit } \\
\hline High sodium & $2.830(1.645-4.868)$ & $<0.001$ & $1.840(0.850-3.987)$ & 0.257 & $3.467(1.896-6.338)$ & $<0.001$ \\
\hline Preserved food & $1.520(0.894-2.584)$ & 0.120 & $1.758(0.860-3.595)$ & 0.123 & $1.393(0.783-2.479)$ & 0.259 \\
\hline Spicy food & $1.309(0.752-2.280)$ & 0.341 & $1.325(0.6 \mid 2-2.872)$ & 0.475 & $1.487(0.8 \mathrm{I} 2-2.722)$ & 0.199 \\
\hline Less fruit & $4.082(2.515-6.625)$ & $<0.001$ & $4.752(2.469-9.145)$ & $<0.001$ & $3.678(2.173-6.225)$ & $<0.001$ \\
\hline \multicolumn{7}{|l|}{ Present relevant illness } \\
\hline $\begin{array}{l}\text { Gastric intestinal metaplasia and } \\
\text { atrophy }\end{array}$ & $15.070(8.999-25.237)$ & $<0.001$ & $16.423(7.513-35.898)$ & $<0.001$ & $14.337(8.102-25.37 \mid)$ & $<0.001$ \\
\hline
\end{tabular}

Abbreviations: GIN, gastric intraepithelial neoplasia; PGIN, proximal GIN; DGIN, distal GIN; OR, odds ratio; 95\% CI, 95\% confidential interval; $\mathrm{Hp}$, Helicobacter pylori.

is one of the important premalignant lesions of GC to some extent. ${ }^{26,27}$ In this study, Hp infection has been identified as an independent risk factor for GIN with an OR of 2.307 (95\% CI: 1.417-3.755). Although some controversies still exist, a growing body of evidence indicated that Hp infection is etiologically related to gastric cancer, and the eradication of $\mathrm{Hp}$ infection could contribute to a reduced incidence of GC. ${ }^{27,28}$ However, the detailed biological mechanisms underlying Hp infection-induced GIN remain far from understood. In addition, we also examined the association between anxiety/ depression status and GIN risk; however, the results were negative. In a Chinese report including 118 patients with gastroesophageal precancerous lesions and 210 healthy controls, it was demonstrated that the anxiety and depression scale scores were higher in patients. ${ }^{29}$

Considering the potential variations between PGIN and DGIN, subgroup analyses were conducted. FCH, less fruit intake, Hp infection, and atrophic gastritis with intestinal metaplasia were proven as the independent risk factors for both PGIN and DGIN. In particular, age was a specific independent risk factor for PGIN while that for DGIN was intake of high sodium. The results suggested that PGIN and DGIN shared similar etiology, similar to PGC and DGC. Further studies are warranted to validate the findings.

This study comprehensively explored the potential risk factors for Chinese GIN patients in the real-world setting and found some positive results. However, some limitations should be acknowledged in interpreting the results. First, the retrospective nature might induce selection bias, even though the study was stringently designed and conducted. Second, the limited sample size in a single institute might reduce the statistical power of the results. Third, the lost follow-up rate was relatively high $(10.5 \%)$, though various efforts have been devoted and the statistical analysis showed balanced in both PGIN and DGIN groups.

\section{Conclusion}

In summary, this study provided a comprehensive overview of the clinical and pathological characteristics of GIN. FCH, high sodium intake, less fruit intake, Hp infection, and atrophic gastritis were identified as the independent risk factors for GIN. Further well-designed, prospective, and unbiased studies with a larger sample size should be conducted to verify our findings.

\section{Disclosure}

The authors report no conflicts of interest in this work.

\section{References}

1. Global Burden of Disease Cancer Collaboration, Fitzmaurice C; Allen C, et al. Global, regional, and national cancer incidence, mortality, years of life lost, years lived with disability, and disability-adjusted life-years for 32 cancer groups, 1990 to 2015: a systematic analysis for the Global Burden of Disease Study. JAMA Oncol. 2017;3(4):524-548.

2. Chen W, Zheng R, Baade PD, et al. Cancer statistics in China, 2015. CA Cancer J Clin. 2016;66(2):115-132.

3. Van Cutsem E, Sagaert X, Topal B, Haustermans K, Prenen H. Gastric cancer. Lancet. 2016;388(10060):2654-2664.

4. Lopez-Basave HN, Morales-Vasquez F, Ruiz-Molina JM, et al. Gastric cancer in young people under 30 years of age: worse prognosis, or delay in diagnosis? Cancer Manag Res. 2013;5:31-36. 
5. Kato M. Diagnosis and therapies for gastric non-invasive neoplasia. World J Gastroenterol. 2015;21(44):12513-12518.

6. de Vries AC, van Grieken NC, Looman CW, et al. Gastric cancer risk in patients with premalignant gastric lesions: a nationwide cohort study in the Netherlands. Gastroenterology. 2008;134(4):945-952.

7. Karimi P, Islami F, Anandasabapathy S, Freedman ND, Kamangar F. Gastric cancer: descriptive epidemiology, risk factors, screening, and prevention. Cancer Epidemiol Biomarkers Prev. 2014;23(5):700-713.

8. Ashktorab H, Kupfer SS, Brim H, Carethers JM. Racial disparity in gastrointestinal cancer risk. Gastroenterology. 2017;153(4):910-923.

9. Cancer Genome Atlas Research Network. Comprehensive molecular characterization of gastric adenocarcinoma. Nature. 2014;513(7517):202-209.

10. Hayakawa Y, Sethi N, Sepulveda AR, Bass AJ, Wang TC. Oesophageal adenocarcinoma and gastric cancer: should we mind the gap? Nat Rev Cancer. 2016;16(5):305-318.

11. Yu X, Hu F, Li C, Yao Q, Zhang H, Xue Y. Clinicopathologic characteristics and prognosis of proximal and distal gastric cancer. Onco Targets Ther. 2018;11:1037-1044.

12. Endoscopic Classification Review Group. Update on the paris classification of superficial neoplastic lesions in the digestive tract. Endoscopy. 2005;37(6):570-578.

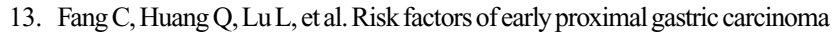
in Chinese diagnosed using WHO criteria. J Dig Dis. 2015;16(6):327-336.

14. Allemani C, Matsuda T, Di Carlo V, et al. Global surveillance of trends in cancer survival 2000-14 (CONCORD-3): analysis of individual records for 37513025 patients diagnosed with one of 18 cancers from 322 population-based registries in 71 countries. Lancet. 2018;391(10125):1023-1075.

15. Sitarz R, Skierucha M, Mielko J, Offerhaus GJA, Maciejewski R, Polkowski WP. Gastric cancer: epidemiology, prevention, classification, and treatment. Cancer Manag Res. 2018;10:239-248.

16. Markowski AR, Markowska A, Guzinska-Ustymowicz K. Pathophysiological and clinical aspects of gastric hyperplastic polyps. World $J$ Gastroenterol. 2016;22(40):8883-8891.

17. Souza RF, Spechler SJ. Concepts in the prevention of adenocarcinoma of the distal esophagus and proximal stomach. CA Cancer J Clin. 2005;55(6):334-351.

18. Xing X, Jia S, Wu J, et al. Clonality analysis of synchronous gastrooesophageal junction carcinoma and distal gastric cancer by wholeexome sequencing. J Pathol. 2017;243(2):165-175.
19. Huang Q, Shi J, Feng A, et al. Gastric cardiac carcinomas involving the esophagus are more adequately staged as gastric cancers by the 7 th edition of the American Joint Commission on Cancer Staging System. Mod Pathol. 2011;24(1):138-146.

20. Suh YS, Han DS, Kong SH, et al. Should adenocarcinoma of the esophagogastric junction be classified as esophageal cancer? A comparative analysis according to the seventh AJCC TNM classification. Ann Surg. 2012;255(5):908-915.

21. Zhao E, Ling T, Xu J, et al. Turning left or right? A comparative analysis in adenocarcinomas of the esophagogastric junction according to the seventh AJCC TNM classification for cancers of the esophagus and stomach: experience in a Chinese single institution. Int J Clin Exp Med. 2015;8(7):10668-10677.

22. Shi J, Sun Q, Xu BY, et al. Changing trends in the proportions of small $(\leq 2 \mathrm{~cm})$ proximal and non-proximal gastric carcinomas treated at a highvolume tertiary medical center in China. J Dig Dis. 2014;15(7):359-366.

23. Huang Q, Fang C, Shi J, et al. Differences in clinicopathology of early gastric carcinoma between proximal and distal location in 438 Chinese patients. Sci Rep. 2015;5:13439.

24. Sedef AM, Kose F, Sumbul AT, et al. Patients with distal intestinal gastric cancer have superior outcome with addition of taxanes to combination chemotherapy, while proximal intestinal and diffuse gastric cancers do not: does biology and location predict chemotherapy benefit? Med Oncol. 2015;32(2):476.

25. Luo G, Zhang Y, Guo P, Wang L, Huang Y, Li K. Global patterns and trends in stomach cancer incidence: age, period and birth cohort analysis. Int J Cancer. 2017;141(7):1333-1344.

26. Venerito M, Link A, Rokkas T, Malfertheiner P. Gastric cancer clinical and epidemiological aspects. Helicobacter. 2016;21(Suppl 1): 39-44.

27. Graham DY. Helicobacter pylori update: gastric cancer, reliable therapy, and possible benefits. Gastroenterology. 2015;148(4):719-731.e3.

28. Lee YC, Chiang TH, Chou CK, et al. Association between Helicobacter pylori eradication and gastric cancer incidence: a systematic review and meta-analysis. Gastroenterology. 2016;150(5):1113-1124.e5.

29. Ma SR, Ma Q, Hao CQ, et al. [Analysis of psychological status and relevant factors of patients with esophageal and gastric cardia precancerous lesions in Linzhou of Henan]. Zhonghua Yu Fang Yi Xue Za Zhi. 2017;51(8):670-674. Chinese. 


\section{Supplementary material}

Table SI Detailed items and the corresponding definitions

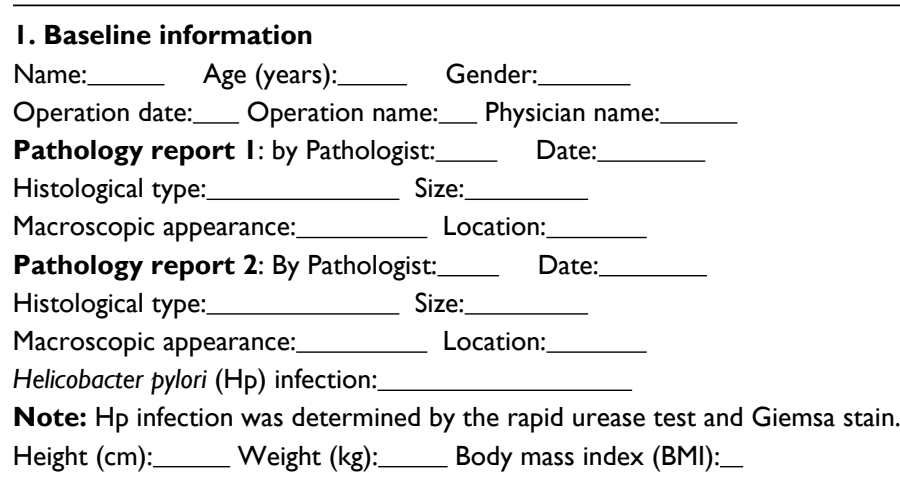

Note: Thin: <18.5; normal: 18.5-24.0; overweight: 24. I-28.0; obesity: $>28.0$.

\section{History of cancer}

$>$ Personal cancer history $(\mathrm{PCH})$ :

$>$ Family cancer history $(\mathrm{FCH})$ :

Note: $\mathrm{FCH}$ includes most common malignancies such as cancers in the gastrointestinal tract, lung, prostate, and breast, etc, except skin basal cell carcinoma in patients' first- and second-degree relatives.

\section{Personal history}

$>$ Occupation:__ History of environmental toxin exposure (ETE):

Note: ETE was defined as a history of direct contact with toxic industrial raw materials or effluent, or living in a radius of $5 \mathrm{~km}$ of a heavily polluted industrious facility, or having a history of occupational toxic chemical exposure over 5 years.

$>$ Alcohol abuse:

Note: It was defined as intake of $500 \mathrm{~mL}$ beer, $250 \mathrm{~mL}$ yellow rice wine, or $50 \mathrm{~mL}$ wine, over twice per week.

$>$ Tobacco abuse:

Note: It was defined as over 10 pack/yr for tobacco abuse.

$>$ Use of nonsteroidal anti-inflammatory drugs (NSAIDs):

Note: It was defined as long-term use due to cardiovascular or rheumatoid diseases for $>6$ months.

\section{Dietary habit}

$>$ Preferences on preserved meat $(\geq 3$ times/wk):

$>$ Preferences on spicy food ( $\geq 3$ times/wk):

$>$ Preferences on smoked food ( $\geq 3$ times/wk):

$>$ Preferences on fried food ( $\geq 3$ times/wk):

$>$ Preferences on hot food ( $\geq 3$ times/wk):

$>$ High sodium intake (defined as over $6 \mathrm{~g}$ daily):

$>$ Intake of fresh fruit and vegetables (occasional, defined as $<2 / \mathrm{wk}$ ):

\section{Present relevant illness}

$>$ Gastroesophageal reflux disease (GERD):

Note: GERD was defined as symptoms of acid reflux such as heartburn and laryngopharyngeal reflux.

$>$ Hypertension ( $>5$ years):

$>$ Diabetes mellitus ( $>5$ years):

$>$ Hiatal hernia (sliding and/or mixed types):

$>$ Anxiety/depression status:

Note: Anxiety status was determined with Zung Self-Rating Anxiety Scale, while depression status was examined with Zung Self-Rating Depression Scale.

Cancer Management and Research

\section{Publish your work in this journal}

Cancer Management and Research is an international, peer-reviewed open access journal focusing on cancer research and the optimal use of preventative and integrated treatment interventions to achieve improved outcomes, enhanced survival and quality of life for the cancer patient. The manuscript management system is completely online and includes

\section{Dovepress}

a very quick and fair peer-review system, which is all easy to use. Visit http://www.dovepress.com/testimonials.php to read real quotes from published authors.

Submit your manuscript here: https://www.dovepress.com/cancer-management-and-research-journal 